\title{
The role of plasma elongation on the linear damping of zonal flows
}

\author{
P. Angelino, ${ }^{1}$ X. Garbet, ${ }^{1}$ L. Villard, ${ }^{2}$ A. Bottino, ${ }^{3}$ S. Jolliet, ${ }^{2}$ Ph. Ghendrih, ${ }^{1}$ V. Grandgirard,${ }^{1}$ \\ B. F. McMillan, ${ }^{2}$ Y. Sarazin, ${ }^{1}$ G. Dif-Pradalier ${ }^{1}$ and T. M. $\operatorname{Tran}^{2}$ \\ ${ }^{1}$ Association Euratom CEA, CEA/DSM/IRFM, Cadarache, 13108 Saint-Paul-lez-Durance, France \\ ${ }^{2}$ CRPP Lausanne, Association Euratom-Confédération Suisse, Switzerland \\ ${ }^{3}$ Max Plank Institut für Plasmaphysik, IPP-EURATOM Association, Garching, Germany
}

(Received 29 January 2008; accepted 25 April 2008; published online 11 June 2008)

\begin{abstract}
Drift wave turbulence is known to self-organize to form axisymmetric macroscopic flows. The basic mechanism for macroscopic flow generation is called inverse energy cascade. Essentially, it is an energy transfer from the short wavelengths to the long wavelengths in the turbulent spectrum due to nonlinear interactions. A class of macroscopic flows, the poloidally symmetric zonal flows, is widely recognized as a key constituent in nearly all cases and regimes of microturbulence, also because of the realization that zonal flows are a critical agent of self-regulation for turbulent transport. In tokamaks and other toroidal magnetic confinement systems, axisymmetric flows exist in two branches, a zero frequency branch and a finite frequency branch, named Geodesic Acoustic Modes (GAMs). The finite frequency is due to the geodesic curvature of the magnetic field. There is a growing body of evidence that suggests strong GAM activity in most devices. Theoretical investigation of the GAMs is still an open field of research. Part of the difficulty of modelling the GAMs stems from the requirement of running global codes. Another issue is that one cannot determine a simple one to one relation between turbulence stabilization and GAM activity. This paper focuses on the study of ion temperature gradient turbulence in realistic tokamak magnetohydrodynamic equilibria. Analytical and numerical analyses are applied to the study of geometrical effects on zonal flows oscillations. Results are shown on the effects of the plasma elongation on the GAM amplitude and frequency and on the zonal flow residual amplitude. (C) 2008 American Institute of Physics. [DOI: 10.1063/1.2928849]
\end{abstract}

\section{INTRODUCTION}

Plasmas in magnetically confined fusion devices are found in turbulent states which enhance the transport of quantities like heat and energy, thus leading to a degradation of the confinement. The turbulence level and the resulting turbulence induced transport depend on nonlinear saturation mechanisms of the underlying instabilities. An essential ingredient of these saturation mechanisms is the generation of large scale flows which behave as regulation agents on the turbulence. Large scale structure generation is known as turbulence self-organization and it is a common phenomenon not only in plasmas, but almost in all forms of turbulence in different media. In toroidal devices, we have the generation of axisymmetric flows, the zonal flows, which present many analogies with the flows observed in planetary atmospheres (Jupiter belts, jet streams). Depending on plasma parameters zonal flows exhibit stationary or oscillatory behavior. These oscillations are induced by the coupling with poloidally asymmetric pressure perturbations. The coupling is due the geodesic curvature and the modes are called Geodesic Acoustic Modes (GAMs). GAMs have been observed on DIII-D (Ref. 1) by Beam Emission Spectroscopy, in JIPT-IIU, ${ }^{2}$ JFT-2M, ${ }^{3}$ and $\mathrm{T}-10,{ }^{4}$ by using heavy ion beam probes, in the ASDEX-Upgrade by Doppler reflectometry ${ }^{5}$ and in the Heliac H1 with an array of Langmuir probes. ${ }^{6}$

The interest in GAMs is due to the role they play in the turbulence saturation mechanism. Indeed, while zonal flows have always a quenching effect on turbulence, GAMs may act as a destabilizing factor. First, oscillating zonal flows are less effective in shearing the radial extended turbulence eddies, as demonstrated by Hahm. ${ }^{7}$ Second, GAMs may provide a mechanism of transfer of energy back from the zonal flows to the drift waves. ${ }^{8}$

The role played by the nonlinear interactions of zonal flows, GAMs and ion temperature gradient driven turbulence on the ion heat transport has been illustrated by global gyrokinetic simulations in Ref. 9 using the code ORB5. ${ }^{10}$ There the total plasma current $I_{P}$ is used as a control parameter of the zonal flow oscillatory behavior. Low $I_{P}$ (hence high safety factor $q_{s}$ ) corresponds to a regime with oscillatory zonal flows and larger ion heat flux than for high $I_{p}$ (low $q_{s}$ ), in which zonal flows are stationary.

The oscillatory behavior of zonal flows is determined by several plasma parameters which influence the linear damping of GAM oscillations. These parameters are mainly the temperature, the safety factor ${ }^{11}$ and the plasma geometry, as shown by Sugama ${ }^{12}$ for helical systems. Experimental evidence of the dependence of the GAM frequency on plasma elongation has been provided by Doppler reflectometry in ASDEX-Upgrade. ${ }^{13}$

The goal of this paper is to establish a simple relationship between plasma elongation and zonal flow oscillation properties. A fluid model is used to derive an expression for the GAM frequency where the plasma elongation appears as an explicit parameter. The obtained relation is compared to the experimental values and to results from numerical gyrokinetic simulations. 
This paper is organized as follows: in Sec. II, a review of the derivation of the GAM dispersion relation based on a fluid model is presented in which plasma geometry comes into play. In Sec. III an analytical approximation to the plasma equilibrium geometry is presented, and based on this analytical description a new dispersion relation is derived in Sec. IV, which includes the effects of plasma elongation. In Sec. V the analytical approximation is compared to a numerical equilibrium and several improvements to the analytical model are suggested. In Sec. VI a numerical model based on gyrokinetic equations is presented. In Sec. VII results from the dispersion relation and from the numerical calculations are presented and compared. Conclusions are given in Sec. VIII.

\section{ANALYTICAL MODEL}

We use the Braginskii equations ${ }^{14}$ in the collisionless limit. Following Ref. 15, linearization of the conventional ion fluid equations (without diamagnetic effects) yields the following set of equations:

$$
\frac{\partial}{\partial t}\left(n_{i}-\rho_{i}^{2} \nabla_{\perp}^{2} \hat{\phi}\right)=-n_{\mathrm{eq}, i} \mathbf{v}_{D} \cdot \nabla\left(\hat{\phi}+\hat{p}_{i}\right)-n_{\mathrm{eq}, i} v_{T i} \nabla_{\|} \hat{v}_{\| i},
$$

$$
\begin{aligned}
& \frac{\partial \hat{v}_{\| i}}{\partial t}=-v_{T i}\left(\nabla_{\|} \hat{\phi}+\nabla_{\|} \hat{p}_{i}\right), \\
& \frac{\partial \hat{p}_{i}}{\partial t}=-\Gamma \mathbf{v}_{D} \cdot \nabla \hat{\phi}-v_{T i} \Gamma \nabla_{\|} \hat{v}_{\| i},
\end{aligned}
$$

where $\hat{\phi}=e \phi / T_{i, \text { eq }}$ is the electrostatic potential. $n_{i}$, $\hat{p}_{i}=p_{i} / p_{i, \text { eq }}, \hat{v}_{\| i} / v_{T i}$ are the perturbed ion density, pressure, and the component of the velocity parallel to the magnetic field lines, the subscript eq denotes the equilibrium quantities, $m_{i}$ is the ion mass, $e$ the electron charge, $\Gamma$ the ratio of specific heats, $\rho_{i}$ the ion Larmor radius, and $v_{T i}=\sqrt{T_{i} / m_{i}}$ is the ion thermal velocity. The drift velocity that appears in Eq. (1c) is defined as $\mathbf{v}_{D}=-\rho_{j} v_{T} B \mathbf{B} \times \nabla\left(1 / B^{2}\right)$. In the following we drop the subscript ${ }^{\prime}$ for the normalized quantities.

The ion density perturbation is constrained by the electro-neutrality condition. Assuming adiabatic response of electrons on magnetic surfaces, it takes the form,

$$
\frac{n_{i}}{n_{i, \mathrm{eq}}}=\frac{e}{T_{e, \mathrm{eq}}}(\phi-\langle\phi\rangle),
$$

where the wedge brackets $\langle\ldots\rangle$ indicate a magnetic surface average. We make the ansatz, $\phi=\phi_{0}+\phi_{1}$, and the same for $v_{\|}$and $p$. Here $\phi_{0}$ is the magnetic surface averaged potential (i.e., $\left.\phi_{0}=\langle\phi\rangle\right)$. The following ordering holds true: $\partial_{t} \sim \rho_{i}, \phi_{1}, v_{\| 1}, p_{1} \sim \rho_{i}, \mathbf{v}_{D} \cdot \nabla \sim \rho_{i}$. At the proper order in $\rho_{i}$, the linearized system of these fluid equations yields

$$
\begin{aligned}
& \partial_{t}\left(\rho_{i}^{2} \nabla_{\perp}^{2} \phi_{0}\right)=\mathbf{v}_{D} \cdot \nabla\left(\phi_{1}+p_{1}\right), \\
& \frac{T_{i}}{T_{e}} \partial_{t} \phi_{1}=-\mathbf{v}_{D} \cdot \nabla \phi_{0}-\frac{v_{T_{i}}}{q_{s} R} V_{\| 1},
\end{aligned}
$$

$$
\begin{aligned}
& \partial_{t} p_{1}=-\Gamma \mathbf{v}_{D} \cdot \nabla \phi_{0}-\Gamma \frac{v_{T_{i}}}{q_{s} R} V_{\| 1}, \\
& \partial_{t} V_{\| 1}=-\frac{v_{T_{i}}}{q_{s} R}\left(\phi_{1}+p_{1}\right)
\end{aligned}
$$

from which the dispersion relation is obtained,

$$
\begin{aligned}
\omega^{2}\left(\rho_{i}^{2} \nabla_{\perp}^{2} \phi_{0}\right)= & (\tau+\Gamma)\left[\mathbf{v}_{D} \cdot \nabla\left(\mathbf{v}_{D} \cdot \nabla \phi_{0}\right)\right. \\
& \left.+\left(\frac{v_{T_{i}}}{q_{S} R}\right)^{2}\left(\rho_{i}^{2} \nabla_{\perp}^{2} \phi_{0}\right)\right] .
\end{aligned}
$$

We can rewrite the Laplacian as

$$
\nabla_{\perp}^{2} \phi_{0}=\frac{1}{\sqrt{g}} \partial_{r} \sqrt{g}|\nabla r|^{2} \partial_{r} \phi_{0} .
$$

In the above, $g$ is the metric tensor of magnetic coordinates $(r, \theta, \varphi)$, where $r$ is a flux surface label, $\varphi$ is the toroidal angle, $\theta$ is a poloidal anglelike coordinate chosen so that magnetic field lines are straight in the $(\theta, \varphi)$ plane. In terms of the representation $\mathbf{B}=I \nabla \varphi+\nabla \psi \times \nabla \varphi$ where $\psi$ is the poloidal flux, the safety factor $q_{s}$ and the poloidal angle $\omega, \theta$ is defined as

$$
\theta=\frac{2 \pi}{q_{s}} \int_{0}^{\omega} \frac{I J_{r \omega \varphi}}{R^{2}} d \omega^{\prime}
$$

where $J_{r \omega \varphi}$ is the Jacobian of $(r, \omega, \varphi)$ coordinates. Considering the variation scale of $\phi_{0}$ faster than the variation scale of the geometry, we can approximate

$$
\begin{aligned}
& \nabla_{\perp}^{2} \phi_{0}=\left\langle|\nabla r|^{2}\right\rangle \partial_{r}^{2} \phi, \\
& \left\langle\left(\mathbf{v}_{D} \cdot \nabla\right)^{2}\right\rangle \phi_{0}=\left\langle\left(\mathbf{v}_{D} \cdot \nabla r\right)^{2}\right\rangle \partial_{r}^{2} \phi_{0} .
\end{aligned}
$$

The GAM dispersion relation takes the form

$$
\begin{aligned}
\omega^{2} \rho_{i}^{2}\left(\left\langle|\nabla r|^{2}\right\rangle \partial_{r}^{2} \phi_{0}\right)= & (\tau+\Gamma)\left[\left\langle\left(\mathbf{v}_{D} \cdot \nabla r\right)^{2}\right\rangle \partial_{r}^{2} \phi_{0}\right. \\
& \left.+\left(\frac{v_{T_{i}}}{q_{s} R}\right)^{2} \rho_{i}^{2}\left(\left\langle|\nabla r|^{2}\right\rangle \partial_{r}^{2} \phi_{0}\right)\right] .
\end{aligned}
$$

To evaluate the effect of the elongation on the GAM frequency, we need to know how this parameter modifies the averaged Laplacian $\left\langle|\nabla r|^{2}\right\rangle$ and drift velocity $\left\langle\left(\mathbf{v}_{D} \cdot \nabla r\right)^{2}\right\rangle$ operators, which appear in the GAM dispersion relation.

\section{A MODEL FOR PLASMA GEOMETRY}

We recall a model for the plasma equilibrium introduced by Connor, ${ }^{16}$ which in the following will be referred to as Culham equilibrium. The magnetic surfaces are approximated by the following set of equations: 


$$
\begin{aligned}
R= & R_{0}-[r-E(r)] \cos \omega-\Delta(r)+T(r) \cos 2 \omega \\
& +P(r) \cos \omega, \\
Z= & {[r+E(r)] \sin \omega+T(r) \sin 2 \omega-P(r) \sin \omega, }
\end{aligned}
$$

$(R, Z, \varphi)$ are the cylindrical coordinates and $R_{0}$ is the plasma major radius. The other parameters $\Delta, E, T$ are related, respectively, to the Shafranov shift, the elongation and the triangularity. $P$ corresponds to a relabelling of the surfaces. In the following we focus on the role of elongation, therefore we set $T=0$. When $r$ is chosen as the minor radius $\rho$, the following expression is obtained for $P$ :

$$
P=\frac{1}{8} \frac{r^{3}}{R_{0}^{2}}+\frac{1}{2} \frac{r}{R_{0}} \Delta-\frac{1}{2} \frac{E^{2}}{r} .
$$

From Eqs. (10a) and (10b), we can evaluate $|\nabla r|^{2}$ and $\sqrt{g}$,

$$
\begin{aligned}
|\nabla r|^{2}= & 1-2 \Delta^{\prime} \cos \theta+2 E^{\prime} \cos 2 \theta+\frac{3}{4}\left(\frac{r}{R_{0}}\right)^{2}+\frac{\Delta}{R_{0}} \\
+ & \frac{\Delta^{\prime 2}}{2}+\frac{E^{\prime 2}}{2}+\frac{3}{2}\left(\frac{E}{r}\right)^{2}, \\
\frac{\sqrt{g}}{r R_{0}}=\left(\frac{R}{R_{0}}\right)^{2}= & {\left[1-2 \frac{r}{R_{0}} \cos \theta+\frac{2 E}{R_{0}} \cos \theta\right.} \\
& \left.-\left(\frac{r^{2}}{2 R_{0}^{2}}+\frac{r}{R_{0}} \Delta^{\prime}+\frac{2 \Delta}{R_{0}}\right)\right] .
\end{aligned}
$$

In the limit of low plasma pressure, the Shafranov shift can be neglected, and $\Delta$ can be set to zero.

\section{THE DISPERSION RELATION INCLUDING ELONGATION EFFECTS}

With the plasma geometry introduced in the previous section, the Laplacian becomes

$$
\left\langle|\nabla r|^{2}\right\rangle=1+\frac{3}{4}\left(\frac{r}{R_{0}}\right)^{2}+\frac{E^{\prime 2}}{2}+\frac{3}{2}\left(\frac{E}{r}\right)^{2} .
$$

Truncating the expansion to first order in $r / R_{0}$, the geometric term in the Laplacian becomes

$$
\left\langle|\nabla r|^{2}\right\rangle=1+\frac{E^{\prime 2}}{2}+\frac{3}{2}\left(\frac{E}{r}\right)^{2}
$$

Let us consider now the drift velocity operator $\left\langle\left(\mathbf{v}_{D} \cdot \nabla r\right)^{2}\right\rangle$. The drift velocity is given by the expression

$$
\begin{aligned}
\mathbf{v}_{D} & =2 \rho_{i} v_{T_{i}} \frac{B_{0} \mathbf{B}}{B^{2}} \times \frac{\nabla B}{B} \\
& =-\rho_{i} v_{T_{i}} B_{0} \mathbf{B} \times \nabla \frac{1}{B^{2}} \\
& =-\rho_{i} v_{T_{i}} \frac{\mathbf{B}}{B_{0}} \times \nabla\left(\frac{B_{0}}{B}\right)^{2} .
\end{aligned}
$$

Approximating $B \simeq\left(B_{0} R_{0}\right) / R$, we can write

$$
\begin{aligned}
\left(\frac{B_{0}}{B}\right)^{2} \simeq\left(\frac{R}{R_{0}}\right)^{2}= & -2 \frac{r}{R_{0}} \cos \theta-\left(\frac{r^{2}}{2 R_{0}^{2}}+\frac{r}{R_{0}} \Delta^{\prime}+\frac{2 \Delta}{R_{0}}\right) \\
& +\frac{2 E}{R_{0}} \cos \theta
\end{aligned}
$$

which we substitute in the expression for the drift velocity,

$$
\begin{aligned}
\mathbf{v}_{D}=- & \rho_{i} v_{T_{i}} \frac{\mathbf{B}}{B_{0}} \times \nabla\left(\frac{B_{0}}{B}\right)^{2} \\
= & -\rho_{i} v_{T_{i}} \frac{\mathbf{B}}{B_{0}} \times \nabla\left[1-2 \frac{r-E}{R_{0}} \cos \theta\right. \\
& \left.-\left(\frac{r^{2}}{2 R_{0}^{2}}+\frac{r}{R_{0}} \Delta^{\prime}+\frac{2 \Delta}{R_{0}}\right)\right] .
\end{aligned}
$$

The gradient in magnetic coordinates is given by

$$
\nabla=\nabla r \partial_{r}+\nabla \theta \partial_{\theta}
$$

recalling that we have set $\Delta=0$, and neglecting $\mathcal{O}\left[\left(r / R_{0}\right)^{2}\right]$,

$$
\mathbf{v}_{D}=2 \rho_{i} v_{T_{i}} \frac{\mathbf{B}}{R_{0} B_{0}} \times\left[\left(1-E^{\prime}\right) \cos \theta \nabla r+(r-E) \sin \theta \nabla \theta\right],
$$

and multiplying by $\nabla r$,

$$
\mathbf{v}_{D} \cdot \nabla r=\frac{2 \rho_{i} v_{T_{i}}}{B_{0} R_{0}}(r-E) \sin \theta(\mathbf{B} \times \nabla \theta) \cdot \nabla r .
$$

Now, $\mathbf{B}=I \nabla \varphi+\psi_{0}^{\prime} \nabla r \times \nabla \varphi$, with $I=B_{0} R_{0}$. Therefore,

$$
\begin{aligned}
& \nabla r \cdot(\mathbf{B} \times \nabla \theta)=\mathbf{B} \cdot(\nabla \theta \times \nabla R)=\frac{B_{0}}{r R_{0}}, \\
& \mathbf{v}_{D} \cdot \nabla r=2 \rho_{i} v_{T_{i}} \frac{(r-E) \sin \theta}{r R_{0}} .
\end{aligned}
$$

Taking the square of Eq. (23) and averaging over $\theta$,

$$
\left\langle\left(\mathbf{v}_{D} \cdot \nabla r\right)^{2}\right\rangle=\frac{2 \rho_{i}^{2} v_{T_{i}}^{2}}{R_{0}^{2}}\left(1-\frac{E}{r}\right)^{2} .
$$

In a first approximation, the shape function $E$ is taken as linear in the minor radius, $E=\left(\kappa_{s}-1\right) r$, where the parameter $\kappa_{s}$ defines the elongation. With this assumption, the Laplacian and the drift velocity operators take the final form,

$$
\begin{aligned}
& \left\langle|\nabla r|^{2}\right\rangle=1+2\left(\kappa_{s}-1\right)^{2}, \\
& \left\langle\left(\mathbf{v}_{D} \cdot \nabla\right)^{2}\right\rangle=\frac{2 \rho_{i}^{2} v_{T_{i}}^{2}}{R_{0}^{2}}\left(2-\kappa_{s}\right)^{2},
\end{aligned}
$$

which we replace in Eq. (9), in order to get the GAM frequency,

$$
\omega^{2}=(\tau+\Gamma) \frac{v_{T_{i}}^{2}}{R_{0}^{2}}\left[\frac{2\left(2-\kappa_{s}\right)^{2}}{1+2\left(\kappa_{s}-1\right)^{2}}+\frac{1}{q_{s}^{2}}\right] .
$$




\section{STUDY OF THE VALIDITY OF THE APPROXIMATION OF THE GEOMETRY}

The validity of the model for plasma geometry is tested comparing the analytical values of the operators $\left\langle|\nabla \Psi|^{2}\right\rangle$ and $\left\langle\left|v_{D} \cdot \nabla \Psi\right|^{2}\right\rangle$ with the values computed by the equilibrium solver code CHEASE, ${ }^{17}$ on the case $q_{s}(\rho=0.5)=1.44$, where $\rho=r / a$. According to this picture, the choice of the elongation function $E(r)=r\left(\kappa_{s}-1\right)$ is quite a rough approximation of an ideal MHD equilibrium, with constant elongation across magnetic surfaces, as can be seen writing it explicitly,

$$
\begin{aligned}
& R=R_{0}-r\left[2-\kappa_{s}\right] \cos \omega, \\
& Z=r \kappa_{s} \sin \omega
\end{aligned}
$$

while the code CHEASE gives an elongation with a profile as a function of the magnetic surface label (Fig. 3, below, dotted lines). Moreover the parameter $\kappa_{s}$ acts to shrink the magnetic surfaces in the $R$ direction while stretching them in the $Z$ direction, with the purpose of keeping the volume enclosed by the magnetic surfaces constant. This is not the same behavior as the elongation parameter considered in the experiment and by the code CHEASE, which we shall simply call $\kappa$. This parameter is defined as the ratio of the plasma height over the plasma width on the equatorial plane. In terms of $\kappa$, an equilibrium is described by the equations

$$
\begin{aligned}
& R=\hat{R}_{0}+\hat{r} \cos (\omega+\delta \sin \omega), \\
& Z=\hat{r} \kappa \sin (\omega),
\end{aligned}
$$

where $\quad \hat{R}_{0} \equiv[R(\omega=0)+R(\omega=\pi)] / 2 \quad$ and $\quad \hat{r} \equiv[R(\omega=0)$ $-R(\omega=\pi)] / 2$ are, respectively, the major and minor geometric radii. Following Ref. 18, we can identify this equilibrium in terms of the parameters in Eqs. (10a) and (10b) with

$$
\begin{aligned}
& \hat{R}_{0}=R_{0}-\Delta-T, \\
& \hat{r}=r-E, \\
& \kappa=\frac{r+E}{r-E}, \\
& \delta=\frac{4 T}{r} .
\end{aligned}
$$

The parameter $\kappa$ acts only to stretch the magnetic surfaces in the $Z$ direction, therefore expanding the volume enclosed by the surface. Figure 1 shows how a circular equilibrium is modified by Eqs. (30) and (31) with $\kappa=1.75$ and by Eqs. (10a) and (10b) with $\kappa_{s}=1.75$. Notice that the last case corresponds to a CHEASE/experimental elongation of $\kappa=7$. Therefore we must be careful comparing analytical results with numerical and experimental ones; the same definition of the elongation parameter must be chosen. Using Eq. (34) we can now rewrite in term of the experimental/CHEASE $\kappa$ the Laplacian,

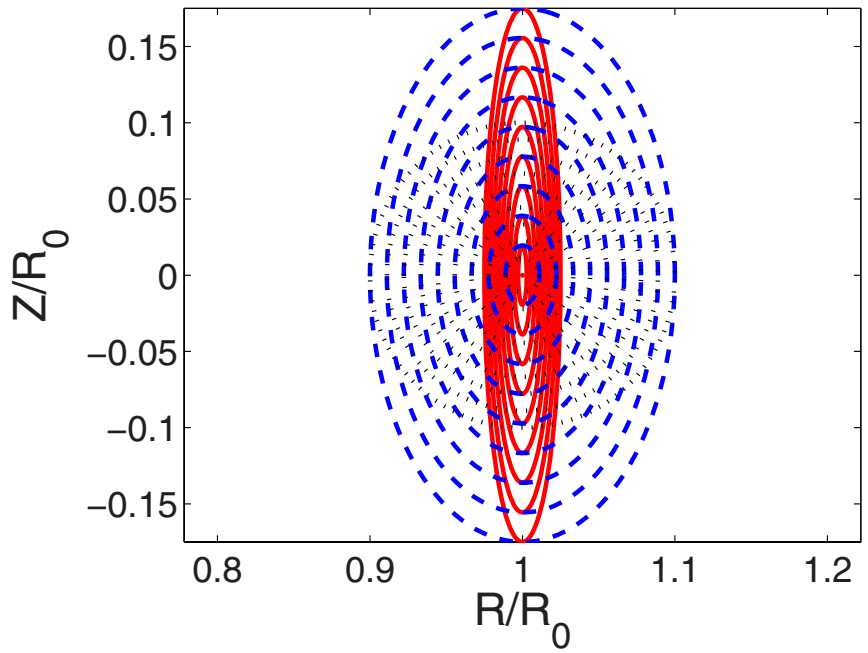

FIG. 1. (Color online) Comparing equilibria. Solid lines correspond to the Culham equilibrium with $\kappa_{s}=1.75$; dashed lines correspond to the experimental-like equilibrium with $\kappa_{b}=1.75$. The starting circular equilibrium is plotted as a reference. The Culham equilibrium has an effective elongation, as defined in the experiment, of $\kappa=7$.

$$
\left\langle|\nabla r|^{2}\right\rangle=\frac{3-2 \kappa+3 \kappa^{2}}{(1+\kappa)^{2}},
$$

and the drift velocity,

$$
\left\langle\left(\mathbf{v}_{D} \cdot \nabla\right)^{2}\right\rangle=\frac{2 \rho_{i}^{2} v_{T_{i}}^{2}}{R_{0}^{2}} \frac{4}{(1+\kappa)^{2}} .
$$

Thus the GAM frequency becomes

$$
\omega^{2}=(\tau+\Gamma) \frac{v_{T_{i}}^{2}}{R_{0}^{2}}\left[\frac{8}{3-2 \kappa+3 \kappa^{2}}+\frac{1}{q_{s}^{2}}\right] .
$$

Now let us go back to the problem of the dependence of the elongation on the magnetic surface, which is so far not correctly included in the model. In addressing this problem analytically, the first step is to have an expression for the radial dependence of $\kappa$. We assume a polynomial dependence which we fit to the CHEASE results $(\rho=r / a$ is the normalized minor radius),

$$
f_{\kappa}(\rho)=a \rho^{3}+b \rho^{2}+c \rho+d .
$$

If the value of the elongation at the plasma edge is $f_{\kappa}(\rho=1)=\kappa_{b}$, from Fig. 3 we observe that at $\rho=0$, elongation is approximately $1+\left(\kappa_{b}-1\right) / 2$, which fixes $d=\left(\kappa_{b}+1\right) / 2$. A good fit is obtained assuming $b=c=0$, thus $a=\left(\kappa_{b}-1\right) / 2$ and the final elongation profile is

$$
f_{\kappa}(\rho)=\frac{1}{2}\left[\left(\kappa_{b}-1\right) \rho^{3}+\kappa_{b}+1\right] .
$$

Remark: In this expression, the plasma boundary elongation $\kappa_{b}$ is a parameter which identifies a whole profile. Introducing $f_{\kappa}$ in Eq. (34), the shaping function $E$ becomes 

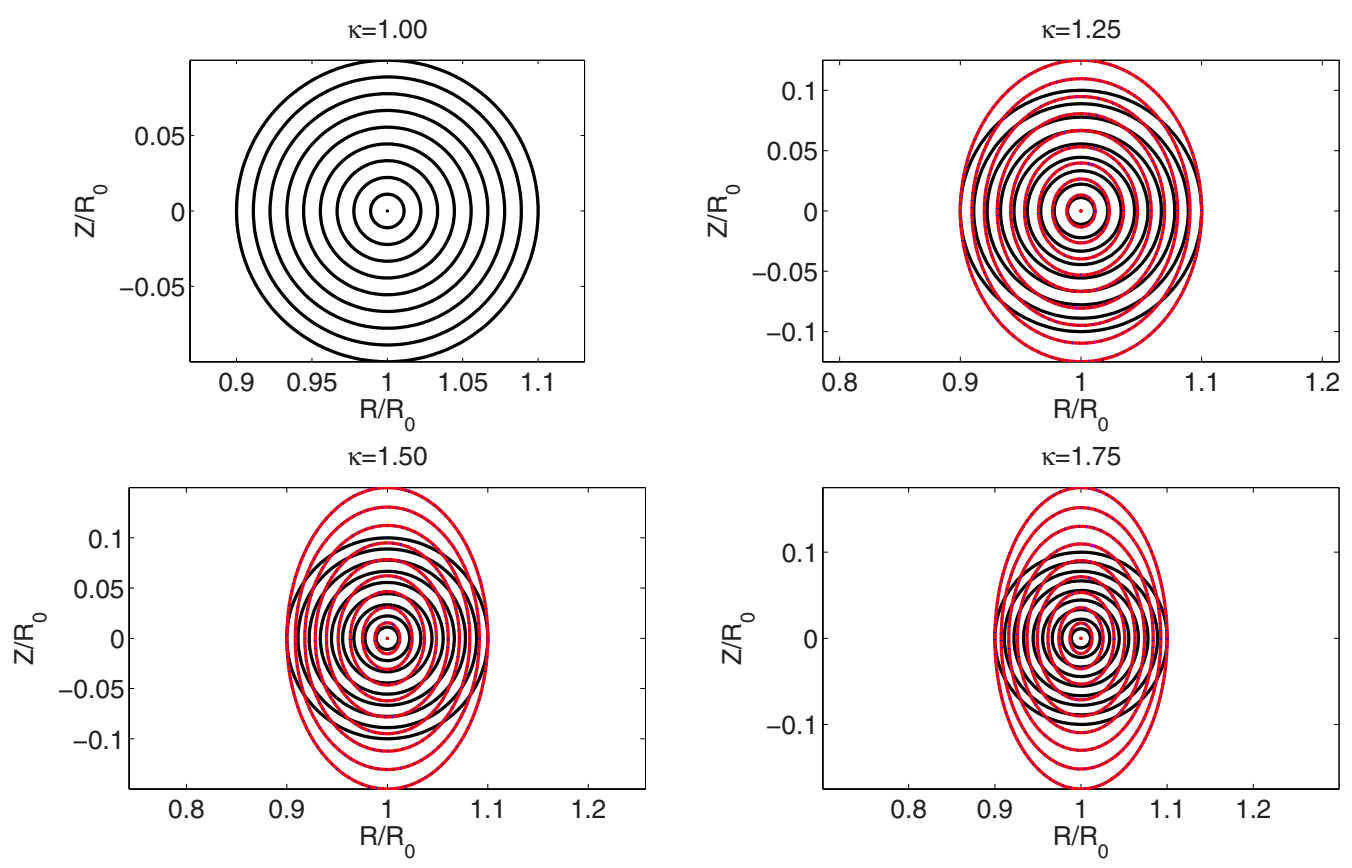

FIG. 2. (Color online) Comparing equilibria. Solid red lines correspond to the adapted Culham equilibrium; the dotted blue lines correspond to the ideal MHD equilibrium from CHEASE. The starting circular equilibrium is plotted as a reference.

$$
E=r \frac{\left(f_{\kappa}-1\right)}{\left(f_{\kappa}+1\right)} .
$$

Plugging this expression into Eqs. (10a) and (10b), it turns out that a shrinking of the minor radius on the equatorial plane is still present,

$$
\hat{r}=r-E=r\left(\frac{2}{f_{\kappa}+1}\right) .
$$

The shrinking may be compensated by defining a rescaled minor radius and shaping function $E$,

$$
\begin{aligned}
& r_{S}=r \frac{\kappa_{b}+1}{2}, \\
& E_{S}=r_{S} \frac{\left(f_{\kappa}-1\right)}{\left(f_{\kappa}+1\right)} .
\end{aligned}
$$

We can now formally write the same equations as Eqs. (10a) and (10b), which now describe an equilibrium where the parameter $\kappa_{b}$ acts as in CHEASE and in the experimental equilibria (see Fig. 2 for a comparison of the plasma shape, and Fig. 3 for the elongation profile),

$$
\begin{aligned}
& R=R_{0}-\left[r_{S}-E_{S}\right] \cos \theta, \\
& Z=\left[r_{S}+E_{S}\right] \sin \theta .
\end{aligned}
$$

With the rescaled $E_{S}$ and $r_{S}$, we write the GAM frequency in the more general form,

$$
\omega^{2}=(\tau+\Gamma)\left(\frac{v_{T_{i}}}{R}\right)^{2}\left[\frac{4\left(1-\frac{E_{S}}{r_{S}}\right)^{2}}{2+E_{S}^{\prime 2}+3\left(\frac{E_{S}}{r_{S}}\right)^{2}}+\frac{1}{q_{S}^{2}}\right]
$$

with

$$
\frac{E_{S}}{r_{S}}=\frac{E}{r},
$$

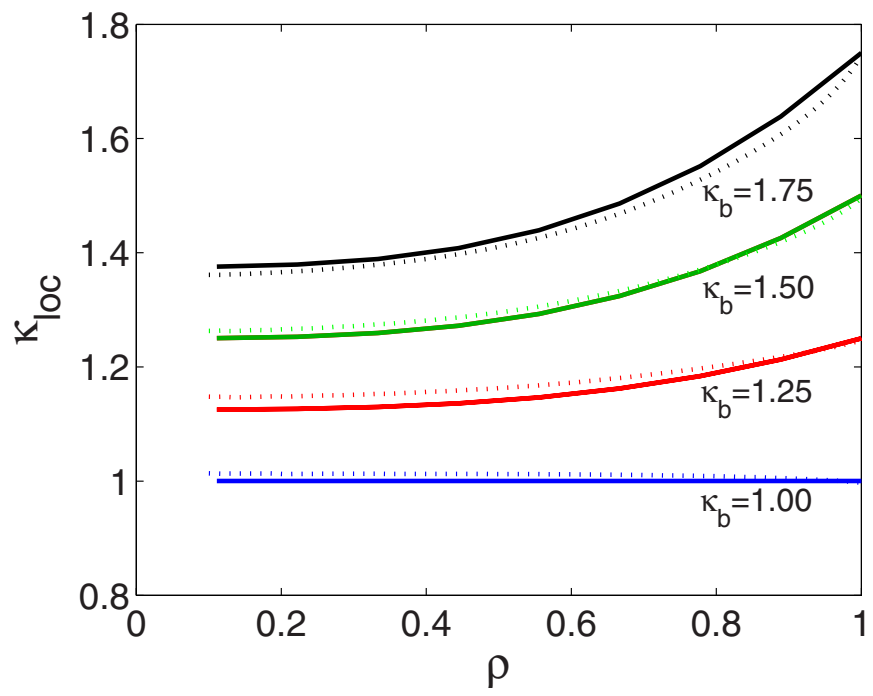

FIG. 3. (Color online) Comparison of the elongation profiles. $\kappa_{\text {loc }}$ is the local value of the elongation, $\rho=r / a$ is the normalized radial coordinate in the equatorial plane. Solid lines are from the adapted analytical Culham equilibrium while the dotted ones are from the CHEASE code. 


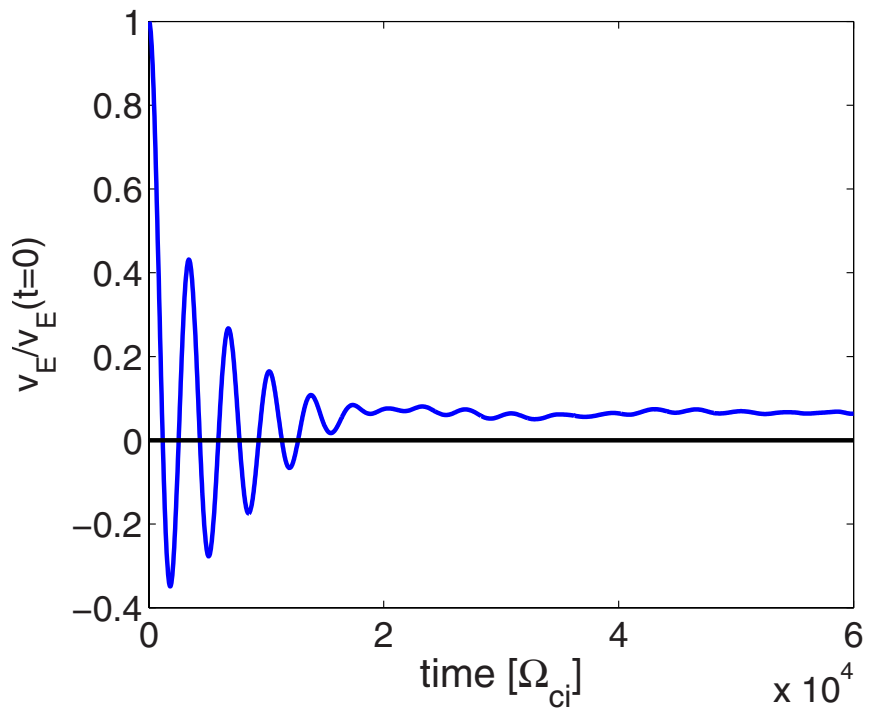

FIG. 4. (Color online) Example of the evolution in time of the poloidal velocity $v_{E}$ for large aspect ratio equilibrium, $\varepsilon=0.1$. The velocity is measured at the radial position $\rho=0.5$, which in this case correspond to a value of the safety factor $q_{s}=1.44$.

$$
E_{S}^{\prime}=\frac{\mathrm{d} E_{S}}{\mathrm{~d} r_{S}}=E_{S}+\frac{r_{S}}{a} \frac{\mathrm{d} f_{\kappa}}{\mathrm{d} \rho} \frac{1}{f_{\kappa}+1}-\frac{r_{S}}{a} \frac{\mathrm{d} f_{\kappa}}{\mathrm{d} \rho} \frac{f_{\kappa}-1}{\left(f_{\kappa}+1\right)^{2}} .
$$

\section{NUMERICAL MODEL}

Simulations are run with the gyrokinetic global code ORB5. ${ }^{19,10}$ The important feature of the code exploited here is the capability to operate using ideal MHD equilibria. The evaluation of the GAM frequency, damping rate, and zonal flows is provided by linear simulations, initialized with an axisymmetric density perturbation. The numerical calculation provides us the time evolution of the electrostatic potential and the poloidal component of the $E \times B$ velocity, which are linearly damped to a residual final value as predicted by the Rosenbluth-Hinton theory. ${ }^{20}$ An example of the output of the ORB5 simulations is plotted in Fig. 4. In this figure the $E \times B$ velocity is plotted normalized to its initial value $v_{E} / v_{E}(0)$ as a function of time. Time is normalized to the ion cyclotron frequency $\Omega_{c i}$.

The basic magnetic configuration is a circular low pressure $\left(\beta \sim 10^{-3}\right)$ plasma. The equilibrium has been supplied by the CHEASE equilibrium code. The major radius is $R=1.32 \mathrm{~m}$. Upon variation of the minor radius $a$, we shall consider two cases of inverse aspect ratio $\epsilon \equiv a / R=0.1$ and $\epsilon=0.36$. Density, ion temperature, and electron temperature profiles are flat with $T_{i}=T_{e}$. The scale of the system is fixed to $L_{x} \equiv 2 a / \rho_{s}=280$, where $\rho_{s}$ is the ion sound Larmor radius. Different safety factor profiles have been used for the test, two of which are plotted in Fig. 5. Four values of the edge elongation, $\kappa_{b}=1,1.25,1.5,1.75$ are considered for studying the dependence of GAM frequency on plasma elongation.

The numerical simulations have been performed with 16 million tracers for $\kappa_{b}=1,1.25$, and 24 million for $\kappa_{b}=1.5,1.75$. More tracers are needed for highly elongated plasma in order to compensate for the increase in plasma volume. The electrostatic potential is solved on a grid

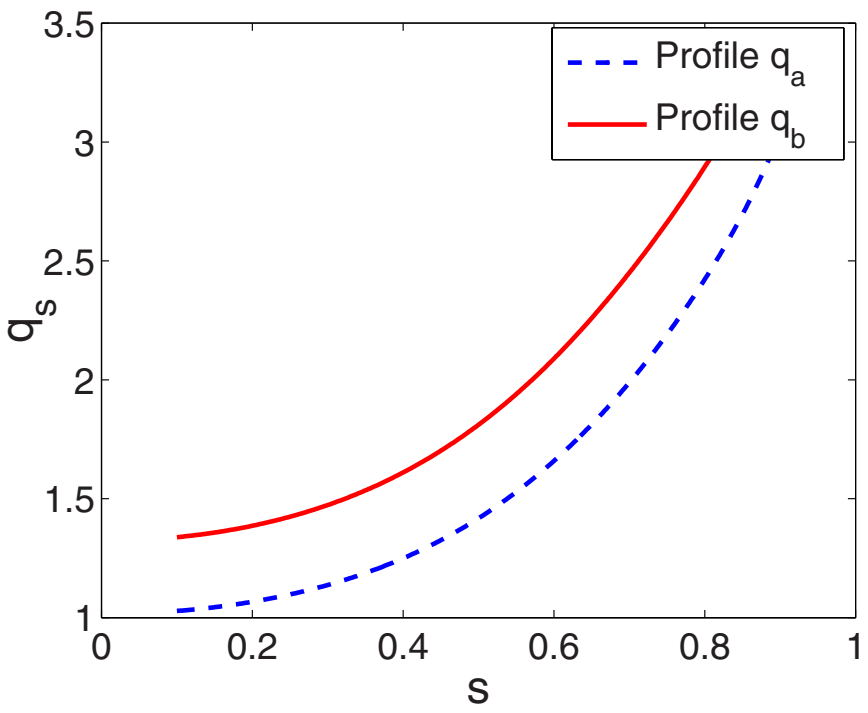

FIG. 5. (Color online) Two safety factor profiles used in the simulations. The lower one takes the value $q_{s}=1.44$ for $s=0.6$ and is named profile $q_{a}$. The higher one takes the value $q_{s}=2.1$ for $s=0.6$ and is named profile $q_{b}$.

$128 \times 128 \times 64$, in toroidal coordinates $s=\sqrt{\psi / \psi_{a}}(\psi$ is the poloidal magnetic flux and $\psi_{a}$ its value at the edge), $\theta$ (straight field line angle), $\varphi$ (toroidal angle). The zonal flow damping test has been performed with the ORB5 code in the linear mode, which means that nonlinear terms in particle trajectory equations have been suppressed. In order to reproduce the results of Hinton and Rosenbluth, we solve only for the $n=0, m=0$ component of the electrostatic potential, the other modes are Fourier filtered. The initial condition has been prepared in order to obtain an axisymmetric ion density perturbation $\delta n_{i}=n_{0} \sin (\pi \rho)$. Here $\delta n_{0}$ is chosen so that $\left\langle v_{E}\right\rangle_{\rho}(t=0)=0.07 v_{T i}$. After a convergence test, a time step of $\Delta t \Omega_{c i}=40$ (where $\Omega_{c i}$ is the ion cyclotron frequency) has been chosen. The ions considered are deuterium.

\section{RESULTS}

Before analyzing the effects of the elongation, we check our model against variation of other parameters, notably the safety factor profile and the aspect ratio.

\section{A. $\omega_{\text {GAM }}$ dependence on the safety factor $q_{s}$}

For a circular plasma, kinetic and fluid theories predict slightly different frequencies. From the kinetic dispersion relation, $\omega_{\mathrm{GAM}}$ is given by ${ }^{12,21}$

$$
\omega_{\mathrm{GAM}}^{2}=\left[\omega_{\mathrm{GAM}, 0}^{2}+\frac{1}{q_{s}^{2} \omega_{\mathrm{GAM}, 0}^{2}}\left(\frac{23}{2}+8 \tau_{e}+2 \tau_{e}^{2}\right)\right]\left(\frac{v_{T_{i}}}{R}\right)^{2},
$$

where $\omega_{\mathrm{GAM}, 0}$ is defined as

$$
\omega_{\mathrm{GAM}, 0}^{2}=\frac{7}{2}+2 \tau_{e} .
$$

From the fluid dispersion relation, ${ }^{22}$ 


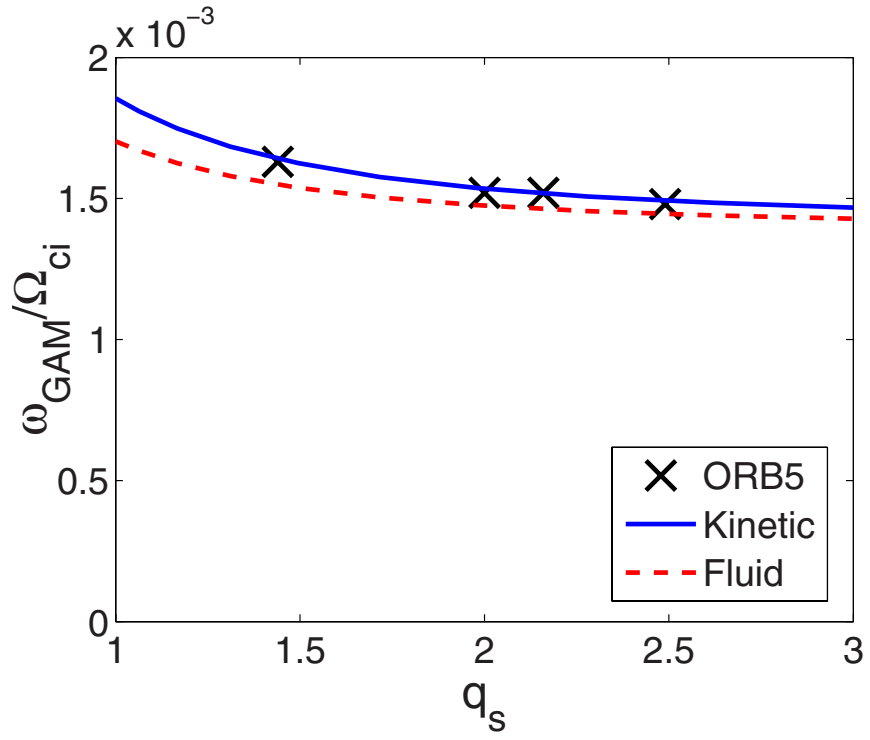

FIG. 6. (Color online) GAM frequency as a function of the safety factor $q_{s}$. Results from kinetic, Eq. (49), and fluid, Eq. (51), dispersion relations are compared with gyrokinetic simulation results, for a value of the inverse aspect ratio $\varepsilon=0.1$ and a radial position $\rho=0.5$. Frequencies are normalized to the ion cyclotron frequency $\Omega_{c i}$.

$$
\omega_{\mathrm{GAM}}^{2}=\left(\Gamma+\tau_{e}\right)\left(2+\frac{1}{q_{S}^{2}}\right)\left(\frac{v_{T_{i}}}{R}\right)^{2} .
$$

In a recent work, ${ }^{23}$ a kinetic-fluid closure model is derived for zonal flows, by which the kinetic $\omega_{\mathrm{GAM}, 0}$ can be recovered. Numerical simulations with ORB5 show a dependence of $\omega_{\mathrm{GAM}}$ on $q_{s}$ in excellent agreement with the kinetic calculation (see Fig. 6). In this comparison, the chosen value of the inverse aspect ratio is $\varepsilon=0.1$ and the radial position is $\rho=r / a=0.5$.

\section{B. Dependence of the GAM damping and frequency on the aspect ratio}

We consider two cases with inverse aspect ratio $\varepsilon=0.1$ (large aspect ratio) and $\varepsilon=0.36$ (cyclone base case ${ }^{24}$ aspect ratio), $q_{s}=2$ at $\rho=r / a=0.5$ in both cases.

The frequency dependence on $\varepsilon$ found in the simulations, Fig. 7, is explained by the dependence in the sound velocity $c_{s} / R$,

$$
\frac{c_{s}}{R}=\varepsilon \frac{2}{L_{x}} \frac{q_{i}}{m_{i}} B_{0} .
$$

The values from kinetic and fluid dispersion relations for $\varepsilon=0.36$ are $\omega_{\mathrm{KIN}} / \omega_{c i}=5.6 \cdot 10^{-3}$ and $\omega_{\mathrm{FL}} / \omega_{c i}=5.4 \cdot 10^{-3}$. Other geometric effects of the aspect ratio [for example, in the Laplacian operator Eq. (14)] are of lower order.

Considering the GAM damping rate $\gamma$, its expression from the kinetic theory is given in Ref. 12. With the present choice of parameters, the radial wave number is small $k_{r} \rho_{s}$ $\sim 0.01$, and the finite orbit width effects can be neglected. In this limit, a simplified formula (Landau damping rate) is found to give a good approximation of the numerical results,

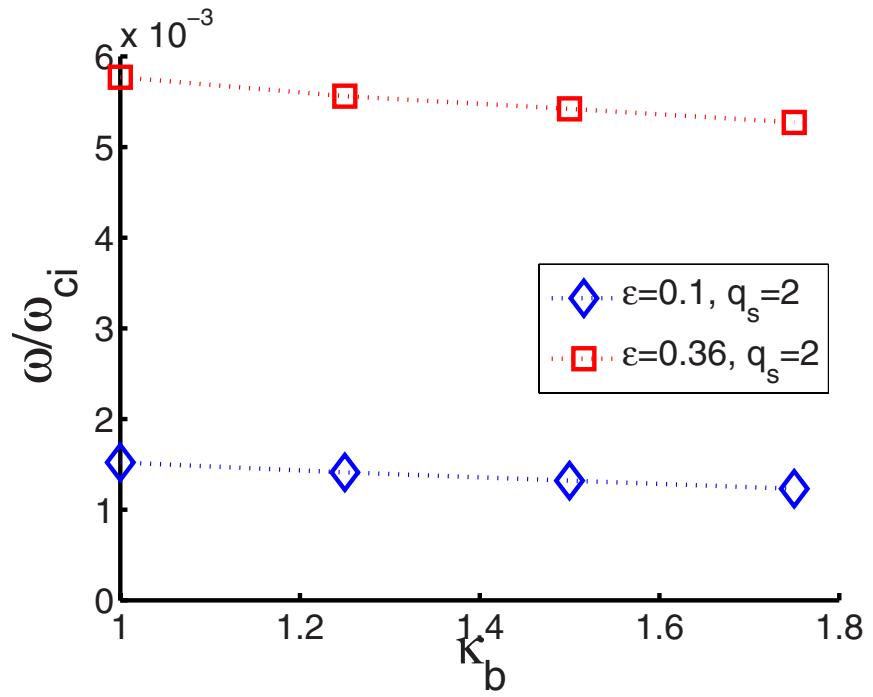

FIG. 7. (Color online) GAM frequency as a function of the edge elongation for two values of the inverse aspect ratio. Results from the gyrokinetic simulations. The dependence on $\kappa_{b}$ is the same for both $\varepsilon$ with a shift explained by the variation of $c_{s} / R$ with the aspect ratio. Frequencies are normalized to the ion cyclotron frequency $\Omega_{c i}$.

$$
\gamma_{\mathrm{KIN}}=\omega_{\mathrm{GAM}} \exp \left(-\hat{\omega}_{\mathrm{GAM}}^{2} / 2\right),
$$

where the normalized GAM frequency is defined as $\hat{\omega}_{\mathrm{GAM}}$ $=\omega_{\mathrm{GAM}} q_{s} R / v_{T i}$. For an inverse aspect ratio of $\varepsilon=0.36$, Eq. (53) gives $\gamma_{\mathrm{KIN}} / \omega_{c i}=3.0 \cdot 10^{-4}$ and for $\varepsilon=0.1, \gamma_{\mathrm{KIN}} / \omega_{c i}$ $=8.1 \cdot 10^{-5}$. From ORB5 (Fig. 8), $\gamma_{0.1} / \omega_{c i}=8 \cdot 10^{-5}$ and $\gamma_{0.36} / \omega_{c i}=2.8 \cdot 10^{-4}$. Again a factor 3.6 between the aspect ratios explains the differences between the corresponding damping rates, and a good agreement between the analytical and the numerical results is found.

The residual zonal flow levels, from ORB5 simulations, are plotted in Fig. 9. In particular, for a circular plasma $\kappa_{b}=1$, the values are $A_{R}=0.030$ for $\varepsilon=0.1$, and $A_{R}=0.053$ for

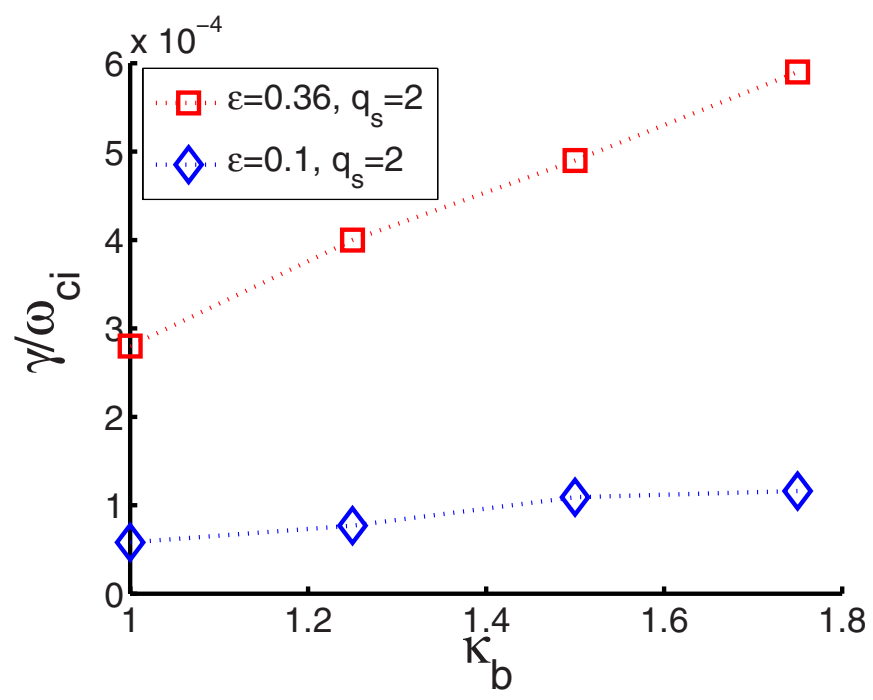

FIG. 8. (Color online) GAM damping rates as a function of the edge elongation for two values of the edge inverse aspect ratio. Results from the gyrokinetic simulations. Growth rates are normalized to the ion cyclotron frequency $\Omega_{c i}$. 


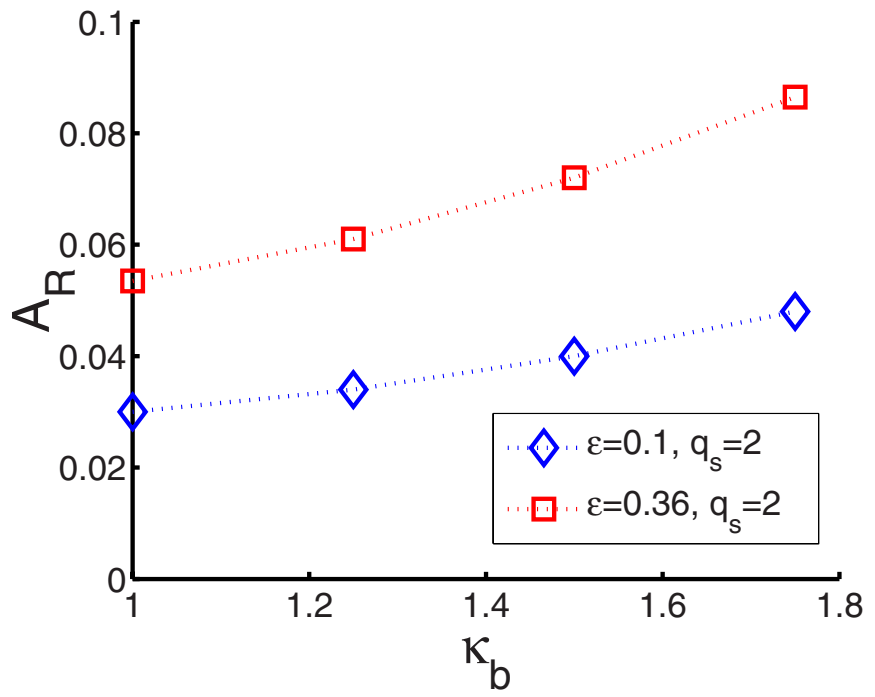

FIG. 9. (Color online) Zonal flow undamped component $A_{R}$ as a function of the edge elongation for two values of the edge inverse aspect ratio. Results from the gyrokinetic simulations.

$\varepsilon=0.36$. The residual zonal flow as given by the RosenbluthHinton theory, ${ }^{20}$

$$
A_{R}=\left[1+1.6 q_{s}^{2}\left(\frac{r}{R}\right)^{-1 / 2}\right]^{-1}
$$

gives $A_{R}=0.034$ for $\varepsilon=0.1$, and $A_{R}=0.062$ for $\varepsilon=0.36$. Corrections of higher order in $\varepsilon$ have been calculated. ${ }^{25}$ Once these corrections are included in Eq. (54), the residuals become $A_{R}=0.030$ for $\varepsilon=0.1$, and $A_{R}=0.050$ for $\varepsilon=0.36$, in very good agreement with ORB5 results (Fig. 9).

\section{C. $\omega_{\text {GAM }}$ dependence on elongation}

In this section the dependence on the elongation of GAM frequency, damping rate, and residual zonal flow component is analyzed in detail. We start comparing the frequency evaluated from the gyrokinetic simulations to the one evaluated with the fluid dispersion relation and the simplified equilibrium $E=r(\kappa-1) /(\kappa+1)$ (for which the elongation is constant across the magnetic surfaces $\left.\kappa=\kappa_{b}\right)$. The expression for $\omega_{\mathrm{GAM}}$ is therefore given by Eq. (38). The results are plotted in Fig. 10. The edge inverse aspect ratio is $\varepsilon=0.1$ and the normalized radial position is $\rho=r / a=0.5$. The analytical and numerical results agree qualitatively. The GAM frequency is reduced with increasing elongation. But the analytical calculation gives a stronger dependence of $\omega_{\mathrm{GAM}}$ on $\kappa$ compared to the numerical simulations. The difference is mainly due to the fact that in the analytical equilibrium $\kappa$ is a global parameter, constant over the whole radius, while in the numerical equilibrium $\kappa$ is a function of the radius. A way to improve the agreement is to plot the frequency $\omega_{\mathrm{GAM}}$ obtained numerically as a function of the averaged elongation $\langle\kappa\rangle$ (Fig. 11), the average being over the full minor radius. In this sense, the value of the elongation to be inserted in the analytical formula in order to get the correct frequency is to be interpreted as an averaged value, because it is then considered constant over the full radius.

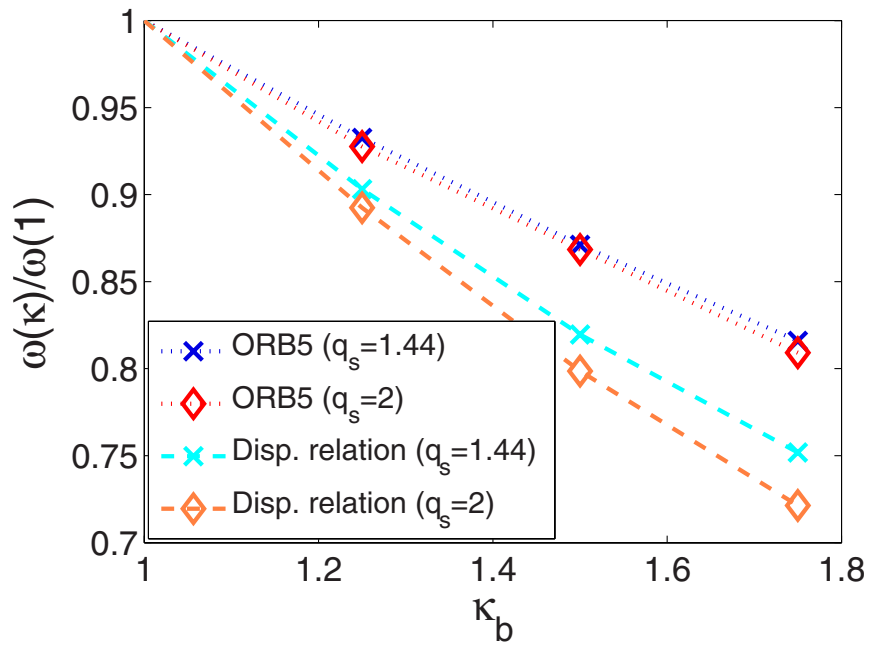

FIG. 10. (Color online) GAM frequency as a function of the edge elongation $\kappa_{b}$. The dotted lines are the results from the numerical simulations, while the dashed lines are the results from the fluid dispersion relation and the equilibrium with constant elongation across the magnetic surfaces. The inverse aspect ratio considered here is $\varepsilon=0.1$ and the frequencies are evaluated at the normalized radial position $\rho=r / a=0.5$. The frequencies are normalized to the value for the circular case $\omega(1)$.

A more rigorous approach is to introduce the equilibrium described by Eqs. (45a) and (45b) where the elongation radial profiles closely reproduce the ones of the ideal MHD equilibria from the code CHEASE, as shown in Fig. 3. The frequency thus obtained is plotted in Fig. 12, and compared with the numerical results, showing a very good agreement. Here choosing the appropriate $\kappa$ for the plot is not an issue, since $\kappa_{b}$ is now a parameter which identifies a profile common to both the numerical and the analytical equilibrium. The "locality" is kept into account choosing the radial position for the measure or evaluation of the frequency.

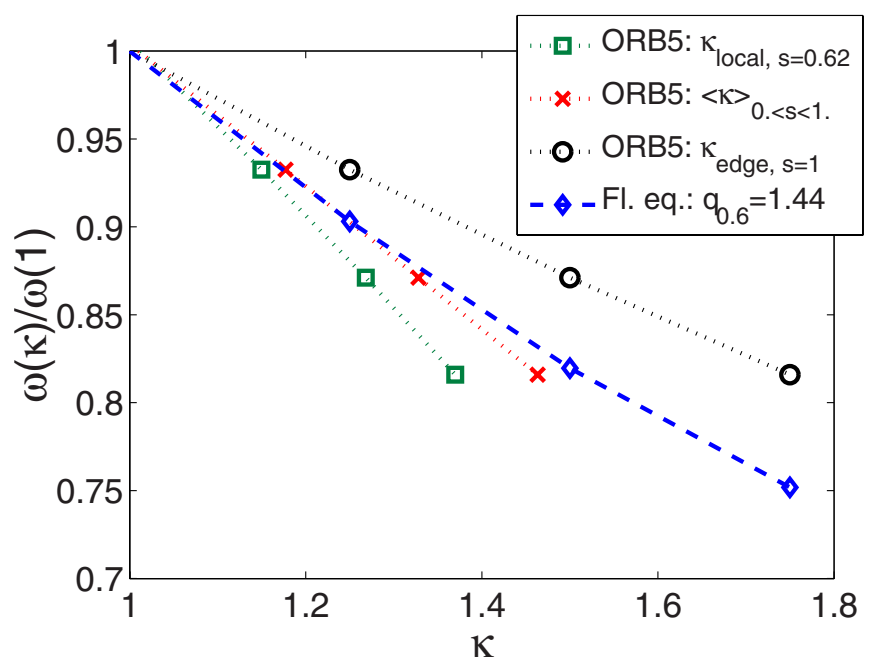

FIG. 11. (Color online) GAM frequency as a function of different definitions of $\kappa$. "Local" means at the radial maximum of the mode, $\rho \sim 0.5,\langle\ldots\rangle$ means a radial average. The better agreement between analytical and numerical results is when the numerical frequency are plotted as a function of $\langle\kappa\rangle$. The edge inverse aspect ratio is $\varepsilon=0.1$ and the frequency are evaluated at the normalized radial position $\rho=r / a=0.5$. The frequencies are normalized to the value for the circular case $\omega(1)$. 


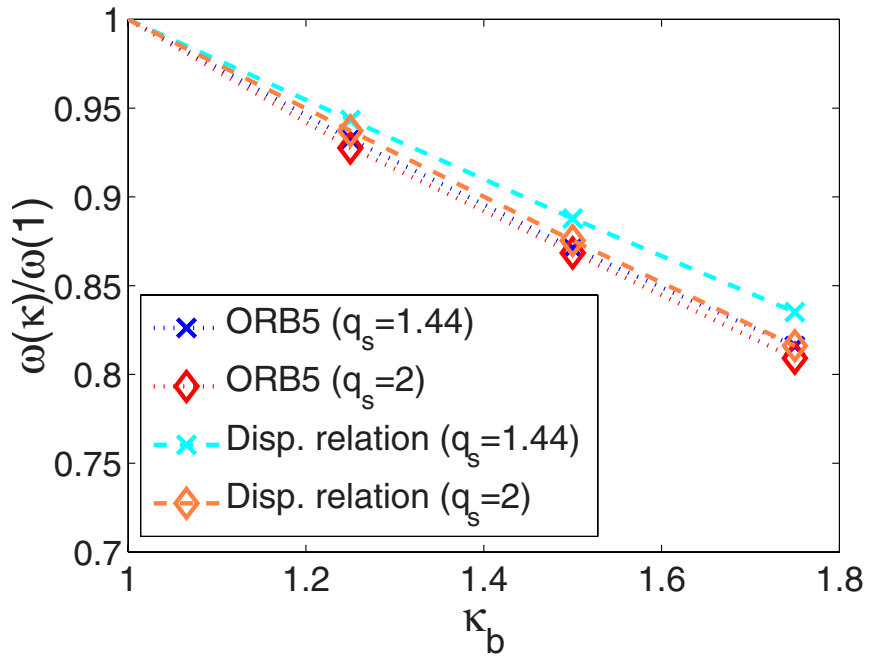

FIG. 12. (Color online) GAM frequency as a function of the edge elongation $\kappa_{b}$. The dotted lines are the results from the numerical simulations, while the dashed lines are the results from the fluid dispersion relation and the modified Culham equilibrium. The inverse aspect ratio considered here is $\varepsilon=0.1$ and the frequency are evaluated at the normalized radial position $\rho=r / a=0.5$. The frequencies are normalized to the value for the circular case $\omega(1)$.

\section{GAM damping rate dependence on elongation}

Linear gyrokinetic simulations show that the GAM damping rate $\gamma$ increases with the plasma elongation. The GAM damping rates are plotted as a function of $\kappa_{b}$ in Fig. 13. Two inverse aspect ratios $(\varepsilon=0.1, \varepsilon=0.36)$ and two safety factor profiles (given in Fig. 5) are considered. The plotted values of $\gamma$ are normalized to the circular case, thus separating the effect of the elongation from the effects of the other parameters. The normalized radial position chosen for measurement is $\rho=r / a=0.5$. For all cases considered here the damping rate $\gamma$ increases with elongation.

We compare these results with the analytical kinetic theory. In Ref. 12 and Eq. (53), $\gamma$ contains a factor

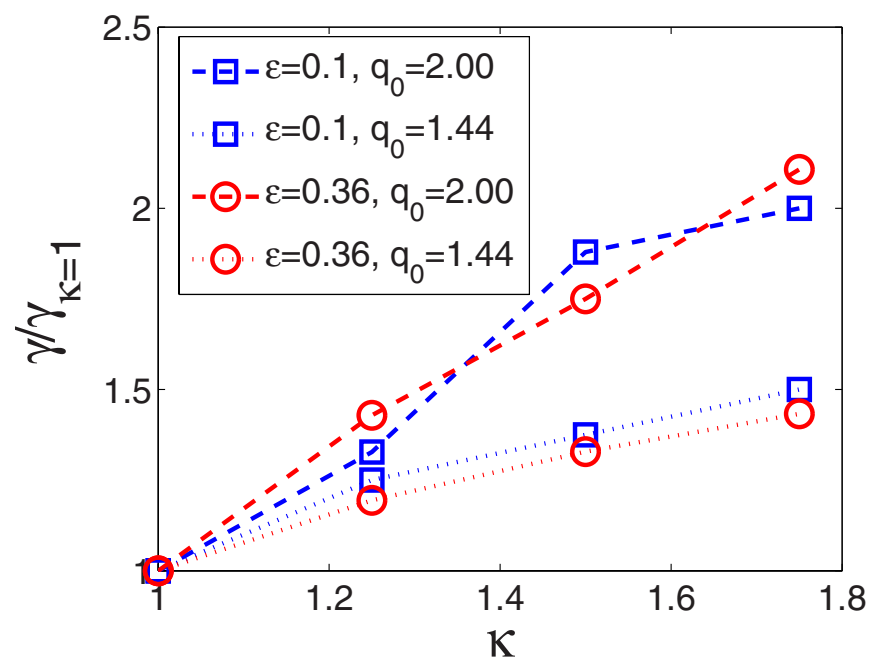

FIG. 13. (Color online) The GAM damping rates as a function of the edge elongation $\kappa_{b}$. Two inverse aspect ratios $(\varepsilon=0.1, \varepsilon=0.36)$ and two safety factor values $\left(q_{s}=1.44, q_{s}=2\right)$ are considered. The damping rates are normalized to the value in the case of a circular plasma $\gamma_{\kappa=1}$.

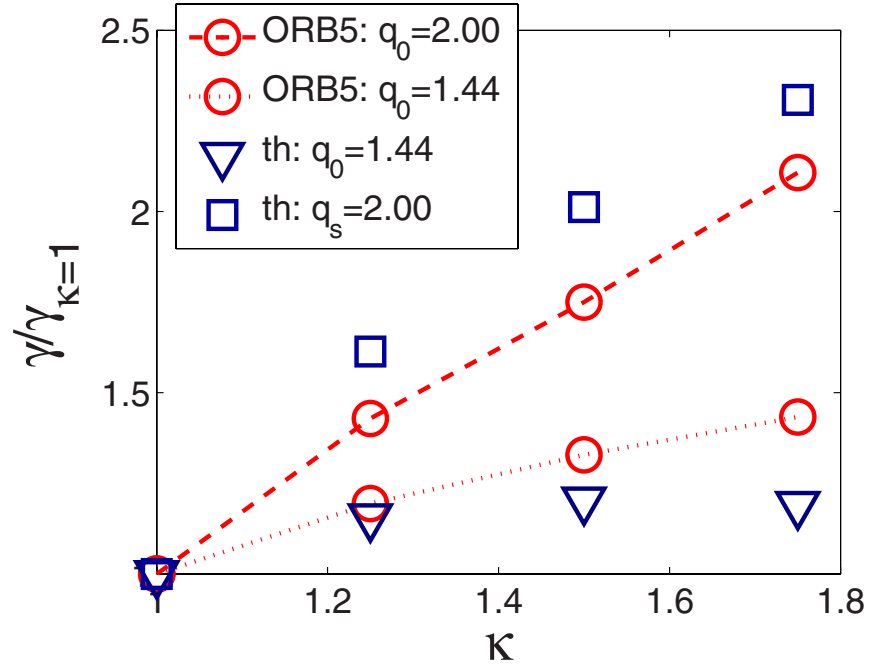

FIG. 14. (Color online) The GAM damping rates as a function of the edge elongation $\kappa_{b}$ from the ORB5 simulation is compared to the one predicted by the analytical calculation. The inverse aspect ratio considered here is $\varepsilon=0.36$. Two values of the safety factor $\left(q_{s}=1.44, q_{s}=2.00\right)$ are considered. The damping rates are normalized to the value in the case of a circular plasma $\gamma_{\kappa=1}$.

$\exp \left(-\hat{\omega}_{\mathrm{GAM}}^{2} / 2\right)$. When the frequency varies the GAM damping rate changes. If we substitute the elongation dependent GAM frequency into the kinetic formula for $\gamma$, we found that most of the dependence of $\gamma$ on $\kappa_{b}$ in Fig. 13 can be explained by this factor (see Fig. 14).

\section{E. Dependence of the residual zonal flow level on elongation}

The residual zonal flow level $A_{R}$ is plotted in Fig. 15 as a function of $\kappa_{b}: A_{R}$ increases with the elongation. As for the damping rates, the values $A_{R}$ are normalized to the circular case, and the normalized radial position is $\rho=r / a=0.5$. The relative variation with the elongation depends weakly on the

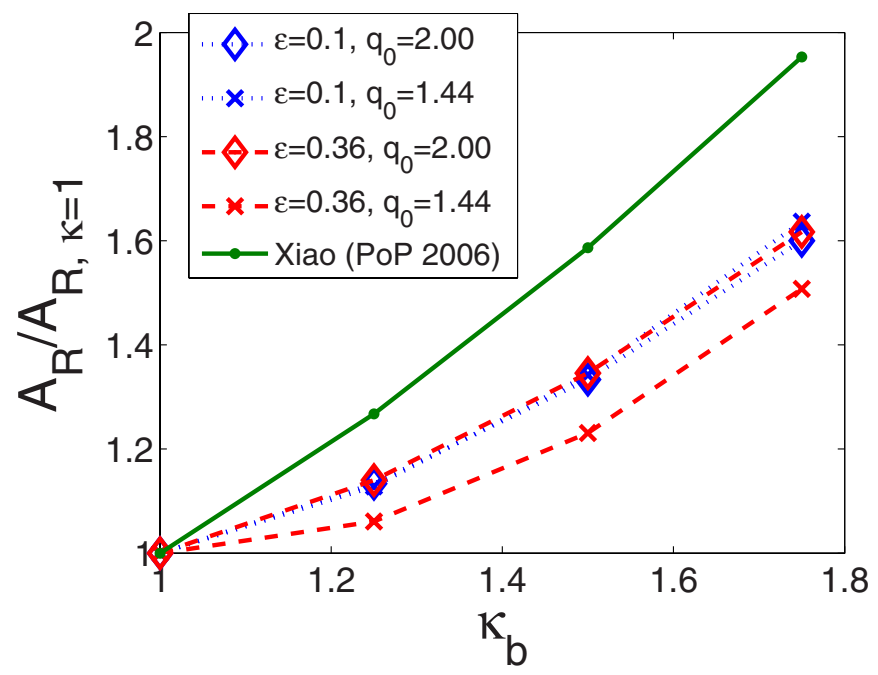

FIG. 15. (Color online) The residual zonal flow level $A_{R}$ as a function of the edge elongation $\kappa_{b}$. Two inverse aspect ratios $(\varepsilon=0.1, \varepsilon=0.36)$ and two safety factor values $\left(q_{s}=1.44, q_{s}=2\right)$ are considered. The analytical value, solid curve, is evaluated for $\varepsilon=0.1$ and $q_{s}=2$. The residuals are normalized to the value in the case of a circular plasma $A_{R, \kappa=1}$. 


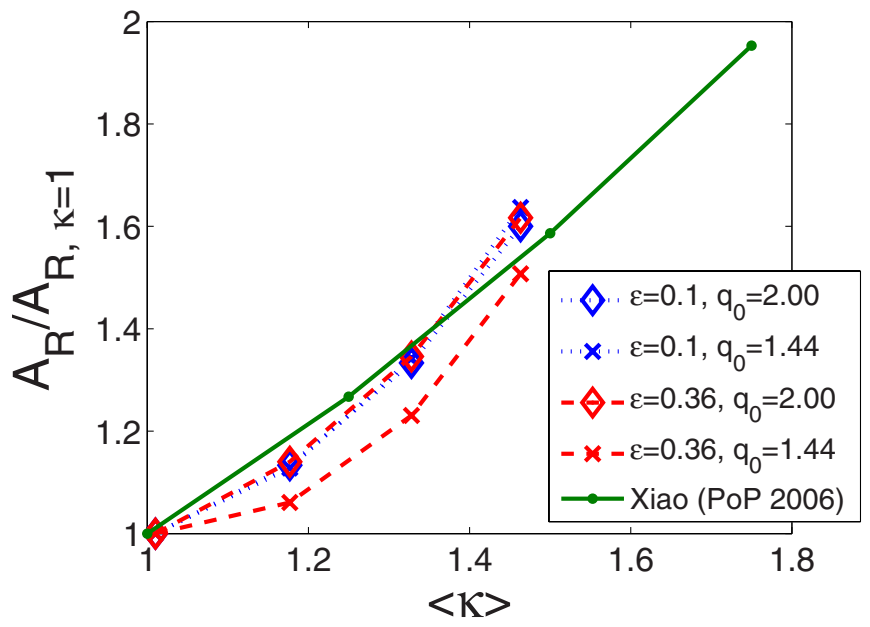

FIG. 16. (Color online) The residual zonal flow level $A_{R}$ as a function of the averaged elongation $\langle\kappa\rangle$. Two edge inverse aspect ratios $(\varepsilon=0.1, \varepsilon=0.36)$ and two safety factor values $\left(q_{s}=1.44, q_{s}=2\right)$ are considered. The analytical value, solid curve, is evaluated for $\varepsilon=0.1$ and $q_{s}=2$. The residuals are normalized to the value in the case of a circular plasma $A_{R, \kappa=1}$.

specific values of both the inverse aspect ratio and the safety factor profile. We compare the values obtained by the gyrokinetic simulations with the expression of the residual given in Ref. 25. In this work, the corrections due to aspect ratio, elongation, triangularity, and Shafranov shift to the $\mathrm{R}-\mathrm{H}$ formula, Eq. (54), are calculated. We already discussed in Sec. VII B the correction of higher order in $\varepsilon$. Here we consider elongation, once again neglecting Shafranov shift and triangularity effects. The equilibrium employed in Ref. 25 is a limit of the Miller equilibrium, ${ }^{26}$ in which the radial variation of the elongation is neglected. Therefore, comparing with the numerical results, we again find a situation similar to the one encountered for the GAM frequency. Comparing the residual as a function of the edge elongation $\kappa_{b}$ (Fig. 15) results in only a qualitative agreement between analytical and numerical values. On the other hand, when the averaged elongation $\langle\kappa\rangle$ is plotted (as in Sec. VII C), a better quantitative agreement is obtained (Fig. 16).

\section{COMPARISON WITH EXPERIMENTAL RESULTS}

We compare the GAM frequency predicted analytically and numerically with the Doppler reflectometry measurement on the ASDEX Upgrade presented by Conway. ${ }^{27}$ Particular care must be taken in this comparison since two branches of GAMs are found experimentally, one localized in the plasma core and one localized in the edge $(\rho \sim 0.95)$. Up to now, only the core branch has been accessible to theoretical analysis, and, unfortunately, this branch has been explored experimentally only at very low elongation, because of technical difficulties. Not all the properties of the two branches are the same. For circular plasmas, the edge GAMs have a higher frequency compared to the core GAMs, whose measured frequency is found to be in good agreement with the theoretical prediction. The measured edge GAM frequency decreases with elongation but the agreement with theory is only qualitative, since the dependence on $\kappa$ found in the experiment is stronger (Fig. 17). No conclusions can

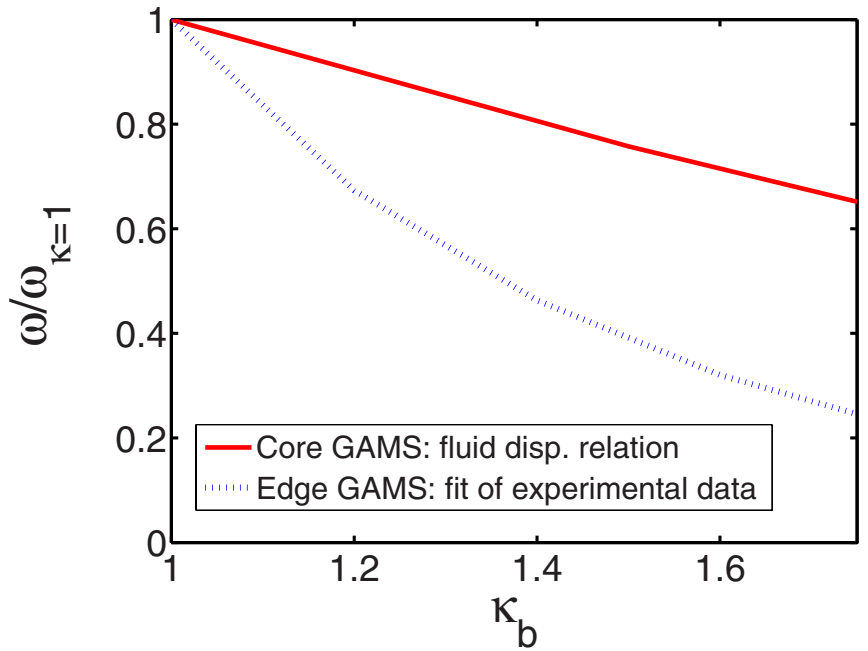

FIG. 17. (Color online) GAM frequency as a function of the edge elongation. The solid line is the curve calculated from the fluid dispersion relation and the dotted line is the fit of the experimental data found in Ref. 27. Particular care must be used when comparing these results, since the fluid dispersion relation is derived for core GAMs, while the experimental data are measured for GAMs localized in the plasma edge.

be drawn for core GAM, since the range of elongation explored is $1<\kappa_{b}<1.16$, which corresponds to a predicted variation of frequency of just $5 \%$.

Several hypotheses could explain the differences. In the experiments the increase in elongation comes with an increase of plasma triangularity. This additional geometry effect is not yet included in the analytical model. For $\kappa_{b}$ larger than 1.4, the experimental plasma configuration changes from limiter to divertor. The divertor configuration has even stronger triangularity, especially close to the separatrix. The presence of a density pedestal and large $k_{r}$ effects (cf. Ref. 12) can contribute to the shift in frequencies.

\section{CONCLUSIONS}

Fluid theory gives a GAM frequency dependence on elongation in good agreement with numerical simulations, once the equilibrium profiles are described with enough accuracy. The equilibrium we proposed was the Culham equilibrium with a radial variation of the elongation which is given by a simple polynomial expression, Eq. (40). A simplified equilibrium can be considered, which neglects the radial variation of the elongation, such as the Culham equilibrium with an elongation function given by $E(r)=r\left(\kappa_{s}-1\right)$, or a Miller equilibrium with constant elongation. But in this case only the dependence on a radially averaged elongation $\langle\kappa\rangle$ can be recovered. The gain in simplicity is paid for by a certain loss in accuracy. The same argument applies to the residual zonal flow level. On the other hand, the analytical kinetic theory calculation ${ }^{12}$ shows that the GAM damping rates $\kappa$-dependence found in the simulations can be explained in good part by $\gamma$ being proportional to $\exp \left(-\hat{\omega}_{\mathrm{GAM}}^{2} / 2\right)$, thus completing a coherent picture of the effects of plasma elongation on the properties of GAMs.

The results presented indicate that the GAM frequency decreases with $\kappa_{b}$, while the GAM damping rate and the 
undamped zonal flow level increase at higher plasma elongations. Therefore, in more elongated plasmas we expect a more effective shearing of the turbulence by larger zonal flows and smaller GAM oscillations. This picture suggests an important impact of elongation on turbulent transport. This hypothesis still needs to be verified by full nonlinear simulations of plasma turbulence.

\section{ACKNOWLEDGMENTS}

We express special thanks to J. D. Conway, J. P. Graves, and G. L. Falchetto for fruitful discussions. The ORB5 simulations have been run on the Pleiades cluster at the EPFL, Switzerland.

The CRPP authors were partly supported by the Swiss National Science Foundation.

${ }^{1}$ G. R. McKee, R. J. Fonck, M. Jakubowski, K. H. Burrell, K. Hallatschek, R. A. Moyer, D. L. Rudakov, W. Nevins, G. D. Porter, P. Schoch, and X. Xu, Phys. Plasmas 10, 1712 (2003).

${ }^{2}$ Y. Hamada, A. Nishizawa, T. Ido, T. Watari, M. Kojima, Y. Kawasumi, K. Narihara, K. Toi, and J.-I. Group, Nucl. Fusion 45, 81 (2005).

${ }^{3}$ T. Ido, Y. Miura, K. Kamiya, Y. Hamada, K. Hoshino, A. Fujisawa, K. Itoh, S.-I. Itoh, A. Nishizawa, H. Ogawa, Y. Kusama, and J.-M. Group, Plasma Phys. Controlled Fusion 48, S41 (2006).

${ }^{4}$ A. V. Melnikov, V. A. Vershkov, L. G. Eliseev, S. A. Grashin, A. V. Gudozhnik, L. I. Krupnik, S. E. Lysenko, V. A. Mavrin, S. V. Perfilov, D. A. Shelukhin, S. V. Soldatov, M. V. Ufimtsev, A. O. Urazbaev, G. V. Oost, and L. G. Zimeleva, Plasma Phys. Controlled Fusion 48, S87 (2006).

${ }^{5}$ G. D. Conway, B. Scott, J. Schirmer, M. Reich, A. Kendl, and the ASDEX Upgrade Team, Plasma Phys. Controlled Fusion 47, 1165 (2005).

${ }^{6}$ M. G. Shats, H. Xia, and M. Yokoyama, Plasma Phys. Controlled Fusion 48, S17 (2006).

${ }^{7}$ T. S. Hahm, M. A. Beer, Z. Lin, G. W. Hammet, W. W. Lee, and W. M. Tang, Phys. Plasmas 6, 922 (1999).
${ }^{8}$ B. Scott, Phys. Lett. A 320, 53 (2003).

${ }^{9}$ P. Angelino, A. Bottino, R. Hatzky, S. Jolliet, O. Sauter, T. M. Tran, and L. Villard, Plasma Phys. Controlled Fusion 48, 557 (2006).

${ }^{10}$ S. Jolliet, A. Bottino, P. Angelino, R. Hatzky, T. Tran, B. Mcmillan, O. Sauter, K. Appert, Y. Idomura, and L. Villard, Comput. Phys. Commun. 177, 409 (2007)

${ }^{11}$ V. B. Lebedev, P. N. Yushmanov, P. H. Diamond, S. V. Novakovskii, and A. I. Smolyakov, Phys. Plasmas 3, 3023 (1996).

${ }^{12}$ H. Sugama and T.-H. Watanabe, Phys. Plasmas 13, 012501 (2006).

${ }^{13}$ G. D. Conway, J. Schirmer, S. Klenge, W. Suttrop, E. Holzhauer, and the ASDEX Upgrade Team, Plasma Phys. Controlled Fusion 46, 951 (2004).

${ }^{14}$ S. I. Braginskii, in Reviews of Plasma Physics, edited by M. A. Leontovitch (Consultant Bureau, New York, 1965), Vol. I, p. 205.

${ }^{15}$ A. B. Hassam and J. F. Drake, Phys. Fluids B 5, 4022 (1993).

${ }^{16}$ J. W. Connor and L. Chen, Phys. Fluids 28, 2201 (1985).

${ }^{17}$ H. Lütjens, A. Bondeson, and O. Sauter, Comput. Phys. Commun. 97, 219 (1996).

${ }^{18}$ J. P. Graves, Phys. Plasmas 12, 090908 (2005)

${ }^{19}$ T. M. Tran, K. Appert, M. Fivaz, G. Jost, J. Vaclavik, and L. Villard, in Theory of Fusion Plasmas, International Workshop (Editrice Compositori, SIF, Bologna, 1999), p. 45.

${ }^{20}$ M. N. Rosenbluth and F. L. Hinton, Phys. Rev. Lett. 80, 724 (1998).

${ }^{21}$ T. Watari, Y. Hamada, T. Notake, N. Takeuchi, and K. Itoh, Phys. Plasmas 13, 062504 (2006).

${ }^{22}$ N. Winsor, J. L. Johnson, and J. M. Dawson, Phys. Fluids 11, 2448 (1968).

${ }^{23}$ H. Sugama, T.-H. Watanabe, and W. Horton, Phys. Plasmas 14, 022502 (2007).

${ }^{24}$ A. M. Dimits, G. Bateman, M. A. Beer, B. I. Cohen, W. Dorland, G. W. Hammett, C. Kim, J. E. Kinsey, M. Kotschenreuther, A. H. Kritz, L. L. Lao, J. Mandrekas, W. M. Nevins, S. E. Parker, A. J. Redd, D. E. Shumaker, R. Sydora, and J. Weiland, Phys. Plasmas 7, 969 (2000).

${ }^{25}$ Y. Xiao and P. J. Catto, Phys. Plasmas 13, 102311 (2006).

${ }^{26}$ R. L. Miller, M. S. Chu, J. M. Greene, Y. R. Lin-Liu, and R. E. Waltz, Phys. Plasmas 5, 973 (1998).

${ }^{27}$ G. D. Conway, C. Tröster, B. Scott, K. Hallatschek, and A. U. Team, in 34th EPS Conference on Plasma Physics, Warsaw, 2007, Europhysics Conference Abstract, Vol. 31F, p. O-4.009 (2007). 